\title{
Special issue on Mexican International Conference on Artificial Intelligence, MICAI 2014 and 2015
}

\author{
Hiram Ponce $^{1}$ - Miguel González-Mendoza ${ }^{2}$ - Ma. Lourdes Martínez-Villaseñor ${ }^{1}$
}

Published online: 17 January 2017

(C) Springer-Verlag Berlin Heidelberg 2017

This special issue of the journal Soft Computing offers extended versions of some of the best-awarded, highreviewed and invited papers presented on the 13th Mexican International Conference on Artificial Intelligence, MICAI 2014, held in Tuxtla Gutiérrez, Chiapas, Mexico, on November 16-22, 2014, under the organization of the Mexican Society for Artificial Intelligence (SMIA) in cooperation with the Instituto Tecnológico de Tuxtla Gutiérrez and Universidad Autónoma de Chiapas, and on the 14th Mexican International Conference on Artificial Intelligence, MICAI 2015, held in Cuernavaca, Morelos, Mexico, on October 2531,2015 , under the organization of the SMIA in cooperation with the Instituto de Investigaciones Eléctricas.

MICAI is an annual conference that disseminates and promotes growth of outstanding research works in all areas of artificial intelligence (AI), including but not limited to: expert systems and knowledge-based systems, knowledge representation and acquisition, multi-agent systems and distributed AI, natural language processing, intelligent interfaces, computer vision, machine learning, pattern recognition, soft computing, reasoning, robotics, planning and scheduling,

Communicated by H. Ponce.

Hiram Ponce

hponce@up.edu.mx

Miguel González-Mendoza

mgonza@itesm.mx

Ma. Lourdes Martínez-Villaseñor

lmartine@up.edu.mx

1 Facultad de Ingeniería, Universidad Panamericana, Campus México, Augusto Rodin 498, 03920 Ciudad de México, Mexico

2 Tecnologico de Monterrey, 14380 México City, Mexico among others. We encouraged both theoretical and practical contributions.

In MICAI 2014 and 2015, we received over 300-400 submissions with an acceptance rate around $25 \%$. At each event, three best papers were awarded. Based on the reviewers' comments from the conference, 12 papers were invited to submit to the special issue after substantial extension. Each submission was reviewed by at least two reviewers in order to guarantee a successful peer-review process expecting originality, quality, correctness and relevance. Finally, we decided to accept nine papers for this special issue.

The selected papers cover different areas of AI, with special emphasis on the integration of theory and applications of the soft computing paradigm, as described following.

In the paper Closed Determination of the Number of Neurons in the Hidden Layer of a Multi-Layered Perceptron Network, Angel Kuri-Morales proposes a new methodology to compute the number of hidden neurons in a multi-layered perceptron (MLP) network by previously conditioning the training data and determining the correct architecture. The experiments show the improvements of the MLP when using this methodology.

Ángel Hernández-Castañeda, Hiram Calvo, Alexander Gelbukh and Jorge J. García Flores assess the effectiveness of support vector networks (SVN) on the task of detecting deception in texts and investigate the possibility degree to build a domain-independent detector using SVN, as described in Cross-Domain Deception Detection Using Support Vector Networks. They discuss the advantages of using SVN on deceptive text classification in different domains.

The paper entitled Segmentation of Carbon Nanotube Images Through an Artificial Neural Network by María Celeste Ramírez, Teresa E. Alarcón, Oscar S. Dalmau and Adalberto Zamudio proposes two segmentation algorithms for carbon nanotube images. The first algorithm uses arti- 
ficial neural networks in the segmentation phase while the second one uses the same supervised method and the relaxed Otsu's threshold. This paper discusses the advantages and limitations of the two proposed algorithms.

In the paper, A Methodology Based on Deep Learning for Advert Value Calculation in CPM, CPC and CPA Networks, Luis Miralles-Pechuán, Dafne Rosso, Fernando Jiménez and Jose M. García propose a methodology for calculating the advertisement value based on different payment methods in online advertising. This proposal uses deep learning to overcome the prediction of the value, and the results are contrasted with traditional machine learning methods.

Miguel Angel Medina-Pérez, Raúl Monroy, J. Benito Camiña and Milton García-Borroto introduce a masquerader detection method, namely Bagging-TPMiner, that is, a oneclass classifier ensemble. In their paper Bagging-TPMiner: A Classifier Ensemble for Masquerader Detection Based on Typical Objects, they present experimental results that show improvements in classification, in contrast to other classifiers, when using the proposed method.

The paper titled Interval Type-2 Fuzzy Logic for Dynamic Parameter Adaptation in the Bat Algorithm by Jonathan Perez, Fevrier Valdez, Oscar Castillo, Patricia Melin, Claudia Gonzalez and Gabriela Martinez proposes an enhancement of the metaheuristic bat algorithm using interval type-2 fuzzy logic aiming to dynamically adapt the inner parameters. Results show that the proposed method outperforms the traditional bat algorithm in a set of benchmark mathematical functions.

In the paper Application of the Distributed Document Representation in the Authorship Attribution Task for Small Corpora, the authors Juan-Pablo Posadas-Durán, Helena
Gómez-Adorno, Grigori Sidorov, Ildar Batyrshin, David Pinto and Liliana Chanona-Hernández propose to use the distributed representation (a technique that allows to represent words in terms of the elements in the neighborhood) at the document level and support vector machines to solve the task of the authorship attribution. Experimental results confirms the usability of this methodology.

Carlos A. Rodriguez, Pedro Ponce and Arturo Molina present an intelligent control strategy for improving versatility on a lower limb exoskeleton. In their paper ANFIS and MPC Controllers for a Reconfigurable Lower Limb Exoskeleton, they compare the adaptive neuro-fuzzy inference system (ANFIS) and the model predictive control (MPC) to determine the best approach for a lower limb exoskeleton.

Finally, the paper A 3-SPS-1S Parallel Robot-Based Laser Sensing for Applications in Precision Agriculture by Ricardo Zavala-Yoe, Ricardo A. Ramírez-Mendoza and Silverio García-Lara presents an intelligent control for a parallel robot for precision agriculture. A closed-loop control system is implemented, using a fuzzy logic controller and an artificial neural network-based model of the robot. Simulation results are discussed.

We thank all the authors for their contributions as well as all the reviewers for their valuable comments. We also thank the editorial team of the journal for their support in the publication of this special issue.

\section{Compliance with ethical standards}

Conflict of interest The authors declare that they have no conflict of interest. 